The University of Southern Mississippi

The Aquila Digital Community

Faculty Publications

$8-1-2020$

\title{
Three-Photon Absorption Spectra and Bandgap Scaling In Direct- Gap Semiconductors
}

\author{
Sepehr Benis \\ University of Central Florida \\ Claudiu M. Cirloganu \\ Lumileds, San Jose, California \\ Nicholas Cox \\ University of Central Florida \\ Trenton Ensley \\ U.S. Army Research Laboratory, Sensors and Electron Devices Directorate \\ Honghua $\mathrm{Hu}$ \\ MKS Instruments
}

See next page for additional authors

Follow this and additional works at: https://aquila.usm.edu/fac_pubs

\section{Recommended Citation}

Benis, S., Cirloganu, C. M., Cox, N., Ensley, T., Hu, H., Nootz, G., Olszak, P. D., Padilha, L. A., Peceli, D., Reichert, M., Webster, S., Woodall, M., Hagan, D. J., Van Stryland, E. W. (2020). Three-Photon Absorption Spectra and Bandgap Scaling In Direct-Gap Semiconductors. Optica, 7(8), 888-889.

Available at: https://aquila.usm.edu/fac_pubs/18536

This Article is brought to you for free and open access by The Aquila Digital Community. It has been accepted for inclusion in Faculty Publications by an authorized administrator of The Aquila Digital Community. For more information, please contact Joshua.Cromwell@usm.edu. 


\section{Authors}

Sepehr Benis, Claudiu M. Cirloganu, Nicholas Cox, Trenton Ensley, Honghua Hu, Gero Nootz, Peter D. Olszak, Lazaro A. Padilha, Davorin Peceli, Matthew Reichert, Scott Webster, Milton Woodall, David J. Hagan, and Eric W. Van Stryland 


\title{
optica
}

\section{Three-photon absorption spectra and bandgap scaling in direct-gap semiconductors}

\author{
Sepehr Benis, ${ }^{1}$ (D) Claudiu M. Cirloganu, ${ }^{2}$ Nicholas CoX,${ }^{1}$ Trenton Ensley,${ }^{3}$ (i) \\ Honghua Hu, ${ }^{4}$ Gero Nootz, ${ }^{5}$ Peter D. Olszak, ${ }^{6}$ Lazaro A. Padilha, ${ }^{7}$ Davorin Peceli, ${ }^{8}$ \\ Matthew Reichert, ${ }^{9}$ (i) Scott Webster, ${ }^{1}$ Milton Woodall, ${ }^{10}$ David J. Hagan,,${ }^{1, *}$ (i] and \\ ERIC W. VAN StryLAND \\ ${ }^{1}$ CREOL, The College of Optics and Photonics, University of Central Florida, Orlando, Florida 32816, USA \\ ${ }^{2}$ Currently at Lumileds, San Jose, California 95131, USA \\ ${ }^{3}$ Currently at CCDC-U.S. Army Research Laboratory, Sensors and Electron Devices Directorate, Adelphi, Maryland 20783, USA \\ ${ }^{4}$ Currently at MKS Instruments, Portland, Oregon 97229, USA \\ ${ }^{5}$ Currently at Division of Marine Sciences, School of Ocean Science and Engineering, University of Southern Mississippi, Stennis Space Center, \\ Mississippi 39529, USA \\ ${ }^{6}$ Currently at Mount Wachusett Community College, Gardner, Massachusetts 01440, USA \\ ${ }^{7}$ Currently at Instituto de Fisica "Gleb Wataghin," Universidade de Campinas, Campinas, 13083-970 Sao Paulo, Brazil \\ ${ }^{8}$ Currently at Extreme Light Infrastructure - Beamlines, FZU AS CR, v.v.i., 25241 Dolni Brezany, Czech Republic \\ ${ }^{9}$ Currently at CACI International Inc., Florham Park, New Jersey 07932, USA \\ ${ }^{10}$ Currently at Leonardo DRS, Dallas, Texas 75251, USA \\ *Corresponding author: Hagan@creol.ucf.edu
}

Received 13 May 2020; revised 20 June 2020; accepted 20 June 2020 (Doc. ID 396056); published 28 July 2020

\begin{abstract}
This paper presents three-photon absorption (3PA) measurement results for nine direct-gap semiconductors, including full 3PA spectra for $\mathrm{ZnSe}, \mathrm{ZnS}$, and GaAs. These results, along with our theory of 3PA using an eight-band Kane model (four bands with double spin degeneracy), help to explain the significant disagreements between experiments and theory in the literature to date. 3PA in the eight-band model exhibits quantum interference between the various possible pathways that is not observed in previous two-band theories. We present measurements of degenerate 3PA coefficients in $\mathrm{InSb}, \mathrm{GaAs}, \mathrm{CdTe}, \mathrm{CdSe}, \mathrm{ZnTe}, \mathrm{CdS}, \mathrm{ZnSe}, \mathrm{ZnO}$, and $\mathrm{ZnS}$. We examine bandgap, $E_{g}$, scaling using -band tunneling and perturbation theories that show agreement with the predicted $E_{g}^{-7}$ dependence; however, for those semiconductors for which we measured full 3PA spectra, we observe significant discrepancies with both two-band theories. On the other hand, our eight-band model shows excellent agreement with the spectral data. We then use our eight-band theory to predict the 3PA spectra for 15 different semiconductors in their zinc-blende form. These results allow prediction and interpretation of the 3PA coefficients for various narrow to wide bandgap semiconductors. $\odot 2020$ Optical Society of America under the terms of the OSA Open Access Publishing Agreement
\end{abstract}

\section{INTRODUCTION}

In the past decades, three-photon absorption (3PA) has shown both fundamental research importance and technological implications in many nonlinear optical (NLO) applications [1-8]. In organic optoelectronics, 3PA can be used as an excitation to observe efficient stimulated emission and frequency upconversion fluorescence emission [2-4]. 3PA can contribute to high-harmonic generation in semiconductor materials $[9,10]$ or may compete with two-photon lasing in direct gap semiconductors [11,12]. Additionally, 3PA in semiconductor quantum dots and nanocrystals has attracted major interest for applications in biolabeling and imaging agents due to the possibility of using longer excitation wavelengths to achieve deeper penetration depths for super-resolution imaging
[1,13-15]. Similarly, 3PA allows the possibility of using light sources in the telecommunication range for optoelectronic applications in wide-bandgap materials such as $\mathrm{ZnO}[5,13,16,17]$. Also, 3PA in semiconductors can be a limiting factor in all-optical switching applications below half the bandgap $[8,18]$. Thus, accurate modeling and experimental verification of 3PA in semiconductors is of great importance for design and characterization of various NLO devices.

While theory and experiment for two-photon absorption (2PA) in direct-gap semiconductors are in excellent agreement [19-27], previous comparisons for 3PA have yielded mixed results [16,20,28-31]. Two convenient scaling rule theories, assuming only two parabolic bands, were developed by Brandi and de Araujo using Keldysh's tunneling approach in 1983 [28,31], and by 
Wherrett using perturbation theory in 1984 [20]. These theories, after reformulating the effective mass, give identical results for 2PA. They also yield similar results for 3PA, differing only in the spectral response and an overall multiplicative constant. Both theories predict that the 3PA coefficient, $\alpha_{3}$, scales with the bandgap energy, $E_{g}$, as $E_{g}^{-7}[20,28,31]$. They find a spectral dependence of $\left(3 \hbar \omega / E_{g}-1\right)^{X} /\left(3 \hbar \omega / E_{g}\right)^{9}$, where tunneling theory yields $X=5 / 2$ and perturbation theory gives $X=1 / 2$. Arbitrary constant scaling factors have been applied to fit measurement results to theory, as was done for 2PA [19,21]. For example, Brandi and de Araujo scaled the calculated 2PA coefficient by an arbitrary factor of six to match data for $\mathrm{CdS}$ [31].

Here, we employ a Kane eight-band model (four bands with double spin degeneracy) [32] to predict the 3PA spectra of 15 direct-gap semiconductors and present Z-scan measurements of the full 3PA spectra of $\mathrm{ZnS}$, and GaAs. In addition, we compare our previous measurement of the 3PA spectrum of $\mathrm{ZnSe}[29,30]$ to the eight-band calculations. Quantum interference between the various pathways available for valence to conduction transitions leads to a more richly featured 3PA spectrum compared to that obtained from simple two-band models. In particular, 3PA spectra show a local peak at photon energies just above $E_{g} / 3$ followed by a relatively flat spectral response through the turn-on of $2 \mathrm{PA}$ at $E_{g} / 2$. A second local maximum may appear between $E_{g} / 3$ and $E_{g} / 2$ depending on the spin-orbit splitting. The data and theory for these three materials are in excellent agreement for the shape of the spectra; however, as mentioned, an overall scaling factor of two to four is needed to match the overall magnitude.

We also performed measurements of $3 \mathrm{PA}$ in $\mathrm{CdS}, \mathrm{ZnO}, \mathrm{ZnTe}$, $\mathrm{CdTe}, \mathrm{CdSe}$, and InSb at selected wavelengths. We show these data along with a few selected literature measurements to demonstrate the $E_{g}^{-7}$ scaling [6,16,33-36]. However, to plot this bandgap scaling, a constant spectral shape must be assumed. This assumption leads to discrepancies for different spectral measurements of a single material because spectral data closely follow the spectra predicted by the eight-band theory. These spectra change from material to material, e.g., the spin-orbit splitting differs for each material. The eight-band theory calculates the 3PA spectra by separately predicting the contributions of all transitions where the heavy-hole (HH), light-hole (LH), and split-off (SO) bands are the initial states. The agreement between our measured spectra and theory for three semiconductors allows us to accurately establish references for predicting and interpreting 3PA spectra for 12 additional zinc-blende semiconductors. Reasonable estimates for materials with different structures could also be made. The role of spin-orbit coupling is crucial in understanding the quantum interference and helps to understand the spectral shape of the 3PA.

\section{EXPERIMENTAL PROCEDURES}

We measured 3PA coefficients using several different pulsed laser systems by employing one of two methods: direct transmittance measurements $[21,37]$ (labeled $T$ in Table 1 ) and open-aperture $Z$-scans [38,39] (labeled $Z$ in Table 1 ) with linearly polarized light. Our Z-scan data in Table 1 was taken with a tunable femtosecond source (Clark MXR 2010 pumped TOPAS-C optical parametric generator/amplifier-OPG/A). Details of the experimental setup are given in $[29,30]$, and an example of data fitting is shown in Fig. 1. The literature data for $\mathrm{As}_{2} \mathrm{Se}_{1.5} \mathrm{~S}_{1.5}$ glass was taken using the same laser system and analyzed in the same way as the femtosecond data in this work [40]. 3PA coefficients for $\mathrm{ZnO}, \mathrm{ZnSe}$, and $\mathrm{CdS}$ at $1064 \mathrm{~nm}$ were found by measuring transmission $(T)$ versus irradiance $(I)$ using 30 ps FWHM pulses from a passively modelocked Quantel Nd:YAG laser. The transmission, $T$, was measured as a function of input irradiance. These values were used to plot $1 / T^{2}$ versus $I^{2}$ which theoretically yields straight lines for constant irradiance in time and space with a slope determining the 3PA coefficient. However, integrals over the Gaussian temporal and spatial coordinates give a slight downward curvature to these lines. The theoretical fits to these curves at low irradiance yield $\alpha_{3}$ [41].

Table 1. 3PA Coefficients [Experimental Data, $\alpha_{3}^{\text {exp }}$, and Values from Eq. (2), Wherrett] with Relevant Material Parameters ${ }^{a}$

\begin{tabular}{|c|c|c|c|c|c|c|c|c|}
\hline Material & $E_{g}[\mathrm{eV}]$ & $E_{p}[\mathrm{eV}]$ & $n$ & $\lambda[\mu \mathbf{m}]$ & $\alpha_{3}^{\exp }$ & Wherrett & $\tau_{p}$ & Method \\
\hline$Z n S^{b}[5]$ & 3.54 & 20.4 & 2.3 & 0.8 & 0.0017 & 0.00130 & $\sim 100 \mathrm{fs}$ & Z \\
\hline $\mathrm{ZnO} \mathrm{O}^{b}[5]$ & 3.27 & 28.2 & 1.95 & 0.9 & 0.0054 & 0.00506 & $\sim 100 \mathrm{fs}$ & $\mathrm{Z}$ \\
\hline $\mathrm{ZnO}$ & 3.27 & 28.2 & 1.94 & 1.06 & 0.022 & 0.0112 & $30 \mathrm{ps}$ & $\mathrm{T}$ \\
\hline $\mathrm{ZnSe}$ & 2.67 & 24.2 & 2.48 & 1.06 & 0.015 & 0.0097 & $30 \mathrm{ps}$ & $\mathrm{T}$ \\
\hline $\mathrm{CdS}$ & 2.42 & 21 & 2.34 & 1.06 & 0.015 & 0.0090 & $30 \mathrm{ps}$ & $\mathrm{T}$ \\
\hline $\mathrm{CdS}$ & 2.42 & 21 & 2.34 & 1.2 & 0.011 & 0.022 & $150 \mathrm{fs}$ & $\mathrm{Z}$ \\
\hline $\mathrm{ZnTe}$ & 2.28 & 19.0 & 2.8 & 1.2 & 0.02 & 0.0196 & $150 \mathrm{fs}$ & $\mathrm{Z}$ \\
\hline $\mathrm{CdSe}$ & 1.90 & 20.0 & 2.5 & 1.5 & 0.24 & 0.085 & $150 \mathrm{fs}$ & $\mathrm{Z}$ \\
\hline CdSe [35] & 1.90 & 20.0 & 2.5 & 1.54 & 0.13 & 0.103 & $\sim 100 \mathrm{fs}$ & $\mathrm{T}$ \\
\hline$A s_{2} S_{1.5} S_{1.5}^{c}[40]$ & 1.74 & 21 & 2.7 & 1.55 & 0.055 & 0.086 & $150 \mathrm{fs}$ & $\mathrm{Z}$ \\
\hline$A l_{0.18} G a_{0.82} A s$ [34] & 1.65 & 21 & 3.34 & 1.55 & 0.05 & 0.045 & $\sim 1 \mathrm{ps}$ & $\mathrm{T}$ \\
\hline $\mathrm{CdTe}$ & 1.44 & 20.7 & 2.7 & 1.75 & 1.2 & 0.19 & $150 \mathrm{fs}$ & $\mathrm{Z}$ \\
\hline$G a A s^{b}[6]$ & 1.42 & 28.9 & 3.4 & 2.3 & 0.35 & 0.82 & $100 \mathrm{fs}$ & $\mathrm{Z}$ \\
\hline $\operatorname{InAs}$ [33] & 0.354 & 21.5 & 3.42 & 9.54 & 1,000 & 11,000 & $\sim 120 \mathrm{ps}$ & $\mathrm{Z}$ \\
\hline $\operatorname{InSb}[36]$ & 0.174 & 23.3 & 3.95 & 10.6 & 200,000 & 18,400 & $45 \mathrm{ps}$ & $\mathrm{T}$ \\
\hline InSb (at $80 \mathrm{~K})$ & 0.228 & 21 & 3.95 & 12 & 25,000 & 46,000 & $10 \mathrm{ps}$ & $\mathrm{Z}$ \\
\hline
\end{tabular}

${ }^{a}$ Materials in italics are taken from the references listed. All $\boldsymbol{\alpha}_{3}$ values are in $\mathrm{cm}^{3} / \mathrm{GW}^{2} . \tau_{p}$ is the pulse width FWHM. The experimental method is either direct transmittance $(\mathrm{T})$ or open-aperture Z-scan $(\mathrm{Z})$. The band parameters used are listed in Table 2.

${ }^{b}$ From the spectra given, the single datum is taken from the region of the graph where the dispersion shown was low.

The bandgap of $\mathrm{As}_{2} \mathrm{Se}_{1.5} \mathrm{~S}_{1.5}(1.74 \mathrm{eV})$ is determined from the linear transmittance spectrum [40]. 

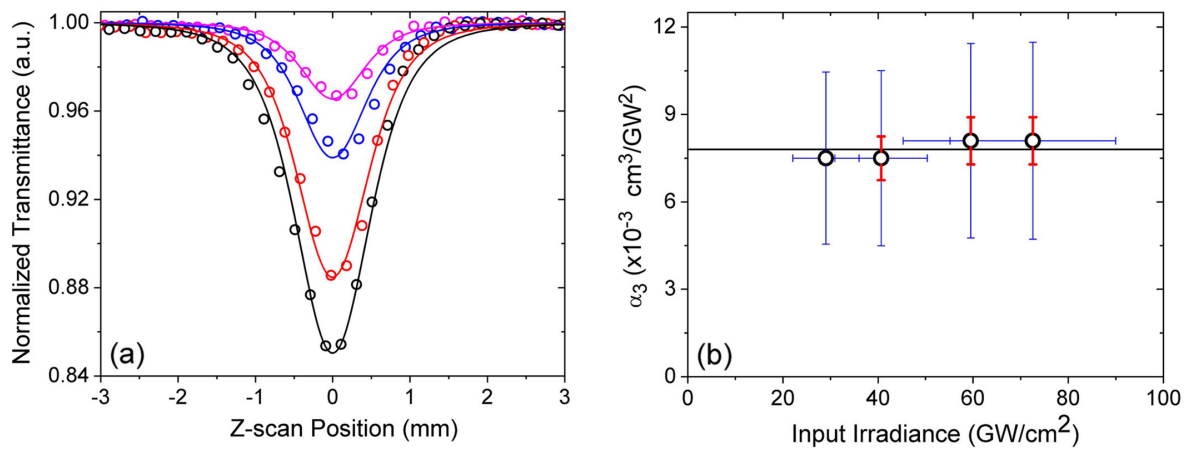

Fig. 1. (a) Z-scans at several peak irradiances in $\mathrm{ZnSe}$ at $1050 \mathrm{~nm}\left(29 \mathrm{GW} / \mathrm{cm}^{2}\right.$ pink, $41 \mathrm{GW} / \mathrm{cm}^{2} \mathrm{blue}, 60 \mathrm{GW} / \mathrm{cm}^{2} \mathrm{red}$, and $\left.72 \mathrm{GW} / \mathrm{cm}^{2} \mathrm{black}\right)$ along with fits (solid lines) using the values for $\alpha_{3}$ shown in (b). (b) $\alpha_{3}$ values obtained from the data in (a). The error bars (blue) are absolute, while the errors (red) relative to the lowest irradiance $Z$-scan are considerably smaller. The average $\alpha_{3}=(7.8 \pm 3) \times 10^{-3} \mathrm{~cm}^{3} / \mathrm{GW}^{2}$ while considering there is no free-carrier absorption.

At high irradiance, effects of FCA increase the losses as discussed below. Measurements on InSb at $12 \mu \mathrm{m}$ were taken using pulses created via difference frequency generation (DFG) of picosecond pulses from a hybrid modelocked Ekspla PL 2143 laser system.

Measurement accuracy of 3PA coefficients decreases with increasing pulse width due to the additional buildup of 3PAexcited carriers. The subsequent free-carrier absorption (FCA) can dominate 3PA for picosecond and nanosecond pulses, and the accompanying free-carrier refraction makes it difficult to collect all the transmitted energy $[42,43]$. For pulses less than $\sim 100 \mathrm{ps,}$ recombination can be ignored so that pulse propagation is simply modeled by considering only $3 \mathrm{PA}$ and FCA [41]. In this model, the change in irradiance induced by $3 \mathrm{PA}$ and FCA is formulated as

$$
\frac{d I}{d z}=-\alpha_{3} I^{3}-\sigma_{\mathrm{FCA}} N I \quad \text { and } \quad \frac{d N}{d t}=\frac{\alpha_{3} I^{3}}{3 \hbar \omega},
$$

where $\alpha_{3}$ is the 3PA coefficient, $\sigma_{\mathrm{FCA}}$ is the FCA cross section, and $N$ is the density of carriers excited via 3PA. These equations are solved numerically for both $\alpha_{3}$ and $\sigma_{\mathrm{FCA}}$ to fit the picosecond Z-scan measurements [44]. In all the measurements reported here, we found FCA to be negligible except for the picosecond Z-scan measurements of GaAs and InSb (80 K). Our T measurements also used $\sigma_{\mathrm{FCA}}=0$.

Data from other references are included in Table 1, distinguished by italicized material names. Material parameters used in the theory discussed in the next section are also given along with the theoretical $\alpha_{3}$ calculated by Wherrett's model. The Keldysh model gives similar results but with somewhat larger discrepancies as explained in Section 3. Broad spectral data taken for $\mathrm{ZnS}, \mathrm{ZnSe}$, and GaAs are shown in Section 4. Values for these spectra are not listed in the table but are included in Fig. 2.

\section{BANDGAP SCALING OF THREE-PHOTON ABSORPTION}

While the eight-band model exhibits the same bandgap scaling as the two-band models, the spectral shape is considerably different making direct comparisons of this scaling problematic. Therefore, we look at using Wherrett's model [20] to demonstrate bandgap scaling. As it turns out, its spectral dependence yields a better fit to the data than that of Brandi and de Araujo. However, as we show below, neither model adequately describes the 3PA spectra. Using a two-parabolic band model, Wherrett's theory gives the 3PA coefficient as

$\alpha_{3}(\omega)=\frac{K_{3} E_{p}^{3 / 2}}{n^{3} E_{g}^{7}} F_{3}\left(\frac{\hbar \omega}{E_{G}}\right) \quad$ where $F_{3}(x)=\frac{(3 x-1)^{1 / 2}}{(3 x)^{9}}$,

where $n$ is the refractive index, and $E_{p}$ is the Kane energy. The Kane energy is a measure of the coupling strength between valence and conduction bands and is about $21 \mathrm{eV}$ for many semiconductors $[20,21,32]$. Both $E_{g}$ and $E_{p}$ have units of eV in Eq. (2), and the constant $K_{3} \cong 25.1 \mathrm{eV}^{11 / 2} \mathrm{~cm}^{3} / \mathrm{GW}^{2}$ is obtained from the two-band theory of Wherrett as estimated by Woodall [20,32,41].

Wherrett's perturbative approach assumes dominance of so-called allowed-allowed-allowed transitions, for which each transition is symmetry-allowed at the Brillouin zone center [20]. On the other hand, the tunneling theory of Brandi and de Araujo gives equivalent results to perturbation theory considering only allowed-forbidden-forbidden transitions [31]. In this case, the forbidden steps are intraband "self" transitions. These two approaches give identical 2PA coefficients because both theories only permit allowed-forbidden combinations in a two-band model. This agreement no longer holds for 3PA because the triply-allowed



Fig. 2. Scaled $3 \mathrm{PA}\left(\alpha_{3}^{\text {scaled }}\right)$ data from literature in Table 1 (stars), picosecond measurements ( $\Delta^{\prime}$ s) [41] and $\Delta$ for InSb at $80 \mathrm{~K}$, and femtosecond data (circles) compared to Wherrett's theory (solid line). Values taken from Table 1, and femtosecond spectra for $\mathrm{ZnSe}$ and $\mathrm{ZnS}$ (every other datum graphed), and GaAs (all fs data graphed) see Figs. 3-5. 
transition pathway is ignored by Brandi and De Araujo, and the allowed-forbidden-forbidden paths are ignored by Wherrett.

Although the data in Table 1 cover many different semiconductors and wavelengths, we can rescale the 3PA coefficients onto a single universal plot by taking advantage of the theory's explicit bandgap dependence; the remaining spectral dispersion is identical for each material in Wherret's model and can be divided out. Labeling the experimentally measured 3PA coefficients as $\alpha_{3}^{\exp }$, we can isolate the bandgap dependence of Eq. (2) by scaling as

$$
\alpha_{3}^{\text {scaled }}\left(E_{g}\right) \equiv \alpha_{3}^{\exp } \cdot \frac{n^{3}}{E_{p}^{3 / 2} F(x)}
$$

The result should be close to the theoretical value of $K_{3} \times E_{g}^{-7}$. Thus, if we plot the experimentally scaled $\alpha_{3}^{\text {scaled }}$ versus $E_{g}$ we expect a simple inverse-seventh power dependence.

The 3PA coefficients from Table 1 are scaled according to Eq. (3) and plotted versus bandgap energy in Fig. 2. We find good overall agreement in the scaling but with notable exceptions. For example, the literature datum for InAs is considerably smaller than predicted. This error may arise because long-wave infrared experiments are much more difficult than those at shorter wavelengths, and the 120 ps pulses used cause further complications from FCA [33]. Also, the value for CdTe is six times larger than theory. Data points at multiple wavelengths are available for GaAs, $\mathrm{ZnSe}$, and $\mathrm{ZnS}$, and their vertical spread demonstrate the inadequacy of Wherrett's spectral function. If Eq. (2) provided an accurate depiction of the frequency scaling, then dividing them out as per Eq. (3) would ensure that all data for a given material lie at the same point. These observations led us to investigate the theory of 3PA using the more sophisticated Kane eight-band model for zinc-blende semiconductors and to measure the full 3PA spectrum of $\mathrm{ZnSe}$, $\mathrm{ZnS}$, and GaAs $[29,30]$. The theory, developed using third-order perturbation theory, is detailed in Appendix A.

It is important to note that both two-band models ignore forbidden interband transitions between hole states that are accounted for in the present work; interactions between $\mathrm{HH}, \mathrm{LH}$, and $\mathrm{SO}$ bands are a crucial component of the 3PA dispersion. Furthermore, including all possible pathways leads to interference effects in the perturbation expansion, so that 3PA cannot even be well-modeled by summing scaled versions of the Wherrett and tunneling theories. Additionally, the eight-band model used here does not include the possibility of polarization anisotropy. The inclusion of higher bands is needed for that [26,27]. However, the magnitude of any possible anisotropy should be less than our experimental errors and is ignored in this paper.

\section{SPECTRAL DEPENDENCE OF THREE-PHOTON ABSORPTION}

A detailed theoretical approach including all possible transitions for the eight-band model was developed and shown to agree with the measured spectral data for $\mathrm{ZnSe}$ [29,30]. Appendix A gives details on that calculation. A comparison of the theoretically predicted 3PA spectrum for polycrystalline $\mathrm{ZnSe}$ (modified from $[29,30])$ is shown in Fig. 3(a) as a function of the three-photon energy sum. The theory is scaled by a factor of 1.9 to better fit the data. The results of similar experiments using femtosecond pulses on polycrystalline $\mathrm{ZnS}$ are shown in Fig. 3(b), where the theory curve is scaled by 2.3. The scaling factor is found via minimizing the sum of square of residuals. We also note that for 2PA the effect of higher conduction bands introduces considerable nonparabolicity into the conduction band, such that 2PA maintains its value at higher photon energies due to the enhanced density-of-states. A similar enhancement in the 3PA which appears to be in accord with the experimental/theory comparison in Fig. 3 should be anticipated. This would also change the overall scaling factor.

These data agree with all the salient features of the new theoretical 3PA spectra. The SO band contribution in $\mathrm{ZnS}$ is not resolved because it lies within the 3PA peak, while it is clearly apparent near $1.2 \mu \mathrm{m}$ in the model for $\mathrm{ZnSe}$. This behavior is expected because the SO energy for $\mathrm{ZnS}, \Delta=0.06$, is small compared to $\Delta=0.42$ for $\mathrm{ZnSe}$ [45].

Having obtained agreement for the wide-gap semiconductors $\mathrm{ZnS}$ and $\mathrm{ZnSe}$, we chose to study the 3PA spectrum of the commonly used narrower bandgap semiconductor GaAs. Published data for the 3PA spectrum includes only four wavelengths, making it difficult to convincingly support any theory [6]. We measured the GaAs ([111] orientation) 3PA spectrum with both picosecond and femtosecond systems. Picosecond measurements of GaAs were made with an Ekspla OPG/A system pumped by a hybrid modelocked Ekspla PL 2143 laser generating pulse widths of approximately 15 ps (FWHM) in the spectral range of 420-2300 nm with linear polarization. FCA contributes significantly to the nonlinear absorption at this pulse width, so Z-scans were performed at several different pulse energies to fit the 3PA and
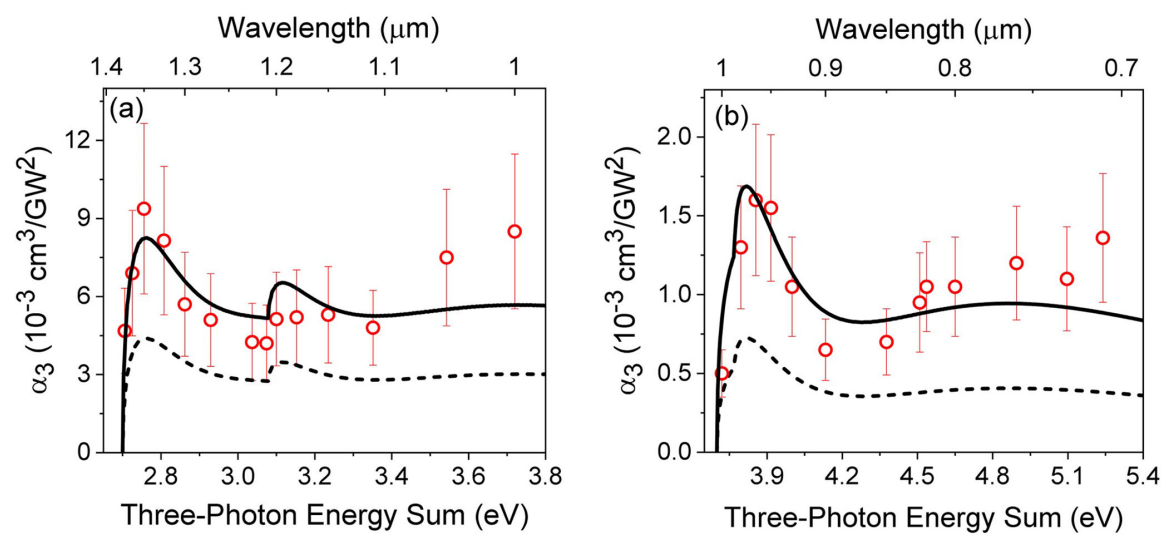

Fig. 3. Measured data (circles) for 3PA in (a) ZnSe (taken from [29,30]) and (b) ZnS obtained from Z-scans, compared to calculated spectra from the eight-band model (black dotted line). Scaling the calculated spectra by factors of 1.9 and 2.3, respectively (solid black curves), provides the best agreement with experimental data. Band parameters used for theoretical calculation are provided in Table 2. 

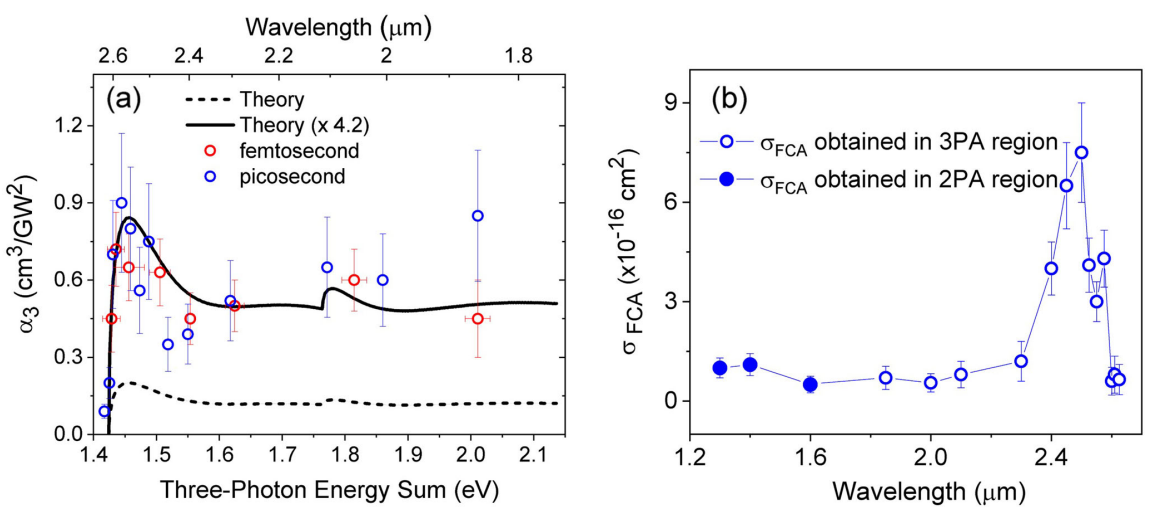

Fig. 4. (a) Measured data for $3 \mathrm{PA}$ in GaAs obtained from Z-scans compared to the calculated spectrum from the eight-band model (black dotted line). Scaling the calculated spectrum by a factor of 4.2 (solid black curve), provides the best agreement with experimental data. Band parameters used for the theoretical calculation are provided in Table 2 . Red circles are experimental data taken with $\sim 150$ ps pulses. Blue circles are data for the $\sim 15$ ps pulses. (b) The FCA cross section from the two-parameter fit for 3PA and the FCA.

FCA coefficients using Eq. (1). The 3PA coefficients obtained are shown in Fig. 4(a) (blue), while the FCA cross sections are shown in Fig. 4(b). The FCA values are in qualitative agreement with those reported in [46].

For femtosecond measurements, we narrowed the laser pulse spectrum with band-pass filters at each wavelength to better resolve the 3PA edge and peak. Again, performing Z-scans, we obtained the data (red) shown in Fig. 4(a). Taking data at several pulse energies did not reveal any FCA, as expected for subpicosecond pulse widths. In the end, we find relatively good agreement between picosecond and femtosecond 3PA measurements. The error bars in both graphs indicate relative errors between measurements, and the overall absolute errors are greater by a factor of 2 .

\section{PREDICTED THREE-PHOTON ABSORPTION SPECTRA AND SPIN-ORBIT COUPLING}

The Kane eight-band model, detailed in Appendix A, is used to predict the 3PA spectra of zinc-blende semiconductors. The only parameters needed are the bandgap, Kane energy, and SO energy, which are compiled in Table 2 for various zinc-blende direct-gap semiconductors. We include a separate column for the ratio $\Delta / E_{g}$ because it turns out to be an important factor in the 3PA spectral shape. This ratio spans from the order of unity in narrow-gap materials to $10^{-2}-10^{-3}$ in wide-gap materials.

Figure 5 gives our predictions of the 3PA spectra for several zinc-blende semiconductors. We explicitly show the contributions from transitions that start in each of the three valence bands: $\mathrm{HH}$, $\mathrm{LH}$, and SO. These bands serve as the initial state "v" in the sumover-states in Eq. (A20). Note that the contribution to the 3PA coefficient from a given initial valence band depends on the behavior of every other band due to the interference of 3PA pathways which pass through each band. However, for brevity we refer to these as the $\mathrm{HH}, \mathrm{LH}$, and $\mathrm{SO}$ contributions.

The graphs of Fig. 5 , in order of increasing $\Delta / E_{g}$, show some general trends. We notice that the $\mathrm{HH}$ (red curves) and LH (blue curves) contributions to $3 \mathrm{PA}$ are nearly identical when $\Delta / E_{g}$ is very small, exemplified by AlN, $\mathrm{ZnO}$, and GaN. 3PA coefficients from both bands exhibit a fast increase near a sum photon energy equal to the bandgap followed by a slow decrease back toward zero as the photon energy increases. The sharp shoulder can be attributed to the dominance of allowed-allowed-allowed transitions,
Table 2. Band-Structure Parameters for Semiconductors in Their Zinc-Blende Form at $300 \mathrm{~K}$ (Except ZnO, at 4.2 K) Used for Modeling of 3PA ${ }^{a}$

\begin{tabular}{ccclccc}
\hline & Material & $\boldsymbol{E}_{\boldsymbol{g}}[\mathbf{e V}]$ & $\boldsymbol{\Delta}[\mathbf{e V}]$ & $\boldsymbol{E}_{\boldsymbol{p}}[\mathbf{e V}]$ & $\boldsymbol{\Delta} / \boldsymbol{E}_{\boldsymbol{g}}$ & Reference \\
\hline $\boldsymbol{\Delta} / \boldsymbol{E}_{\boldsymbol{g}}>1$ & $\mathrm{InSb}$ & 0.174 & 0.81 & 23.3 & 4.65 & {$[32,47]$} \\
& $\mathrm{InAs}$ & 0.354 & 0.39 & 21.5 & 1.10 & {$[47]$} \\
& $\mathrm{GaSb}$ & 0.726 & 0.80 & 22.0 & 1.10 & {$[48,49]$} \\
\hline & $\mathrm{InP}$ & 1.344 & 0.11 & 20.4 & 0.08 & {$[50,51]$} \\
& $\mathrm{GaAs}$ & 1.42 & 0.34 & 28.9 & 0.24 & {$[47,52]$} \\
& $\mathrm{CdTe}$ & 1.44 & 0.91 & 20.7 & 0.63 & {$[45]$} \\
& $\mathrm{CdSe}$ & 1.90 & 0.42 & 20.0 & 0.22 & {$[21,45]$} \\
& $\mathrm{ZnTe}$ & 2.28 & 0.92 & 19.0 & 0.40 & {$[21,45]$} \\
& $\mathrm{CdS}$ & 2.42 & 0.07 & 21.0 & 0.029 & {$[53,54]$} \\
& $\mathrm{ZnSe}$ & 2.67 & 0.42 & 24.2 & 0.16 & {$[21,55]$} \\
\hline \multirow{5}{*}{$\boldsymbol{E}_{\boldsymbol{g}} \ll 1$} & $\mathrm{GaN}$ & 3.24 & 0.017 & 25.0 & 0.005 & {$[47]$} \\
& $\mathrm{ZnO}$ & 3.44 & 0.0087 & 21.0 & 0.002 & {$[56]$} \\
& $\mathrm{ZnS}$ & 3.54 & 0.06 & 20.4 & 0.017 & {$[21,55]$} \\
& $\mathrm{AlP}$ & 3.56 & 0.07 & 17.7 & 0.02 & {$[47]$} \\
& $\mathrm{AlN}$ & 4.90 & 0.02 & 27.1 & 0.001 & {$[45,47]$} \\
\hline
\end{tabular}

"The first three rows indicate materials where the $\mathrm{SO}$ band energy is relatively large $\left(\Delta / E_{g}>1\right)$ and the last five rows indicate materials where the SO band energy is relatively small $\left(\Delta / E_{g} \ll 1\right)$ as discussed in detail below.

${ }^{b}$ Table 2 is sorted based on the values of $E_{g}$. However, note that CdS belongs to the $\Delta / E_{g} \ll 1$ section.

'Eight-band model parameters for $\mathrm{ZnO}$ are given for $\mathrm{T}=4.2 \mathrm{~K}$ (liquid helium temperature).

and interference effects are not readily apparent. In contrast, the SO (green) contribution has the same shoulder, but it is followed by a clear dip from destructive interference. As the photon energy increases, the $\mathrm{SO}$ contribution for $\Delta / E_{g} \geq 0.3$ starts to dominate so that the overall 3PA dispersion stays relatively flat through the 2PA edge.

As $\Delta / E_{g}$ increases, the $\mathrm{LH}$ and $\mathrm{HH}$ contributions develop different spectral shapes. The $\mathrm{HH}$ contribution maintains the shape it had with small spin-orbit coupling, while the LH contribution develops clear markers of destructive interference. The shape of the SO contribution also adjusts slightly as the additional influence from other quantum pathways reduces the net destructive interference. As seen in $\mathrm{CdTe}, \mathrm{GaSb}, \mathrm{InAs}$, and $\mathrm{InSb}$, there is no longer an SO contribution occurring below the 2PA edge when $\Delta / E_{g}>0.5$. 

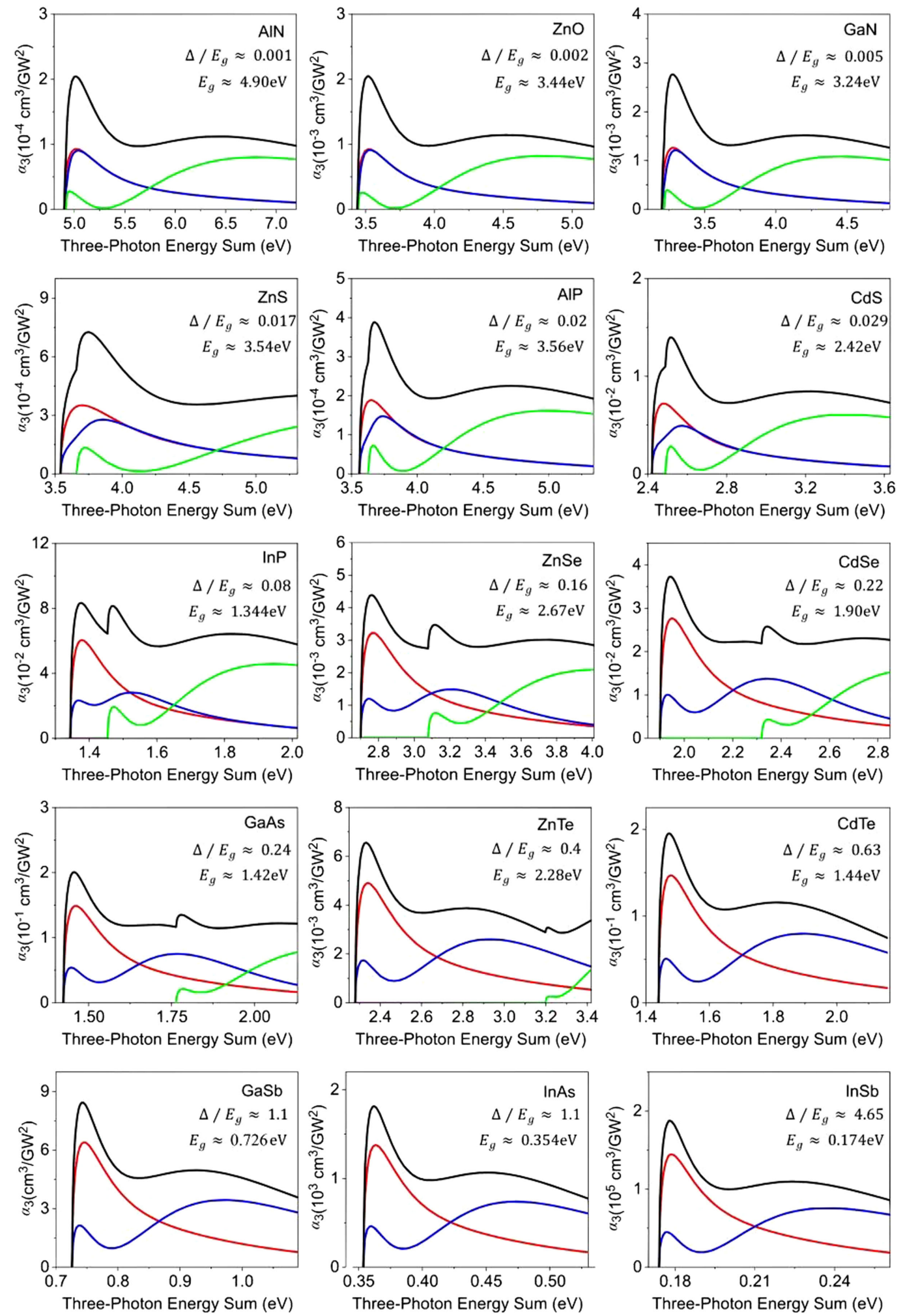

Fig. 5. Theoretical contributions of starting in the $\mathrm{HH}$ (red), LH (blue), and SO bands (green) to the total (black) 3PA in several zinc-blende semiconductors using the eight-band model (see the appendices). These are ordered from small to large $\Delta / E_{g}$ to show the continuous evolution of the 3PA spectral shape, and the 3PA energy sum starts just below the onset of 3PA and goes to where 2PA turns on. No scaling is applied. Band parameters are summarized in Table 2.

In Fig. 6, we fix all band structure parameters except $\Delta$ and show the dependence of the normalized $\alpha_{3}$ on $\Delta / E_{g}$ to investigate the effects of spin-orbit coupling. As $\Delta / E_{g}$ decreases, the $S O$ contribution becomes increasingly important in determining the peak
3PA coefficient and overall spectral shape. However, it is important to note that even where the SO contribution is small, the effect of spin-orbit coupling is still important in determining the shapes of the $\mathrm{HH}$ and $\mathrm{LH}$ contributions. The effect of spin-orbit interaction 


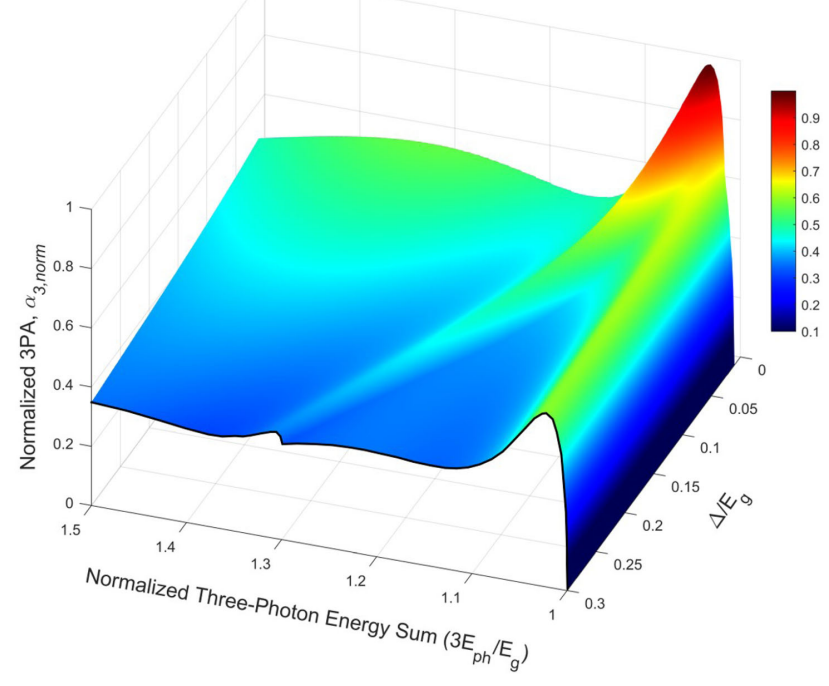

Fig. 6. Dependence of $\alpha_{3, \text { norm }}$ on the normalized three-photon energy sum and $\Delta / E_{g} . \alpha_{3, \text { norm }}$ is normalized to the value of 3PA when $\Delta=0$. The range of $\Delta / E_{g}$ is limited to $\Delta / E_{g}<0.3$ to better represent the effects of the SO band on the 3PA spectra.

on 3PA is described more thoroughly in Appendix B. There, we also give more mathematical detail on the band structure features that give the $\mathrm{HH}, \mathrm{LH}$, and $\mathrm{SO}$ contributions their spectral shape.

\section{CONCLUSIONS}

Prior to this work, the theoretical 3PA bandgap and spectral scaling had not been satisfactorily confirmed by experiment, and conflicting results existed in the literature. We measured 3PA in nine semiconductors, confirming the expected bandgap scaling of $E_{g}^{-7}$ as predicted by simple two-band theories as well as our eight-band theory. Equation (2) (2-band theory of Wherrett [20]) gives the correct scaling and predictions within factors of a few for most of these materials but does not give the correct spectra. On the other hand, the eight-band analysis presented here accurately models 3PA spectra of $\mathrm{ZnSe}, \mathrm{ZnS}$, and GaAs as confirmed by our measurements. The agreement between our measured and calculated spectra shows the value of modeling with more sophisticated band structure; we are then able to predict with confidence the 3PA dispersions of 15 zinc-blende semiconductors for which little data exist. While this theory only strictly applies to zinc-blende crystals, we expect it to give reasonable predictions for materials with other symmetry such as $\mathrm{As}_{2} \mathrm{Se}_{1.5} \mathrm{~S}_{1.5}$ glass.

Our analysis, based on an eight-band model, separately yields the contributions to the 3PA spectra where the initial state is in the $\mathrm{HH}, \mathrm{LH}$, and SO bands. It is important to account for quantum interference between different pathways to predict the spectral dependence of 3PA, and the crucial importance of spin-orbit coupling in determining the spectral shape becomes apparent. The predictions made here can be extremely useful in the case of low-dimensional systems such as quantum wells, quantum dots, nanocrystals, and two-dimensional semiconductors where spin-orbit coupling and intersubband transitions are present and may significantly alter the 3PA process via quantum interference between different bands [57-61].

\section{APPENDIX A: THEORETICAL APPROACH FOR CALCULATING 3PA USING THE KANE EIGHT-BAND MODEL}

In this appendix, we provide detail about the theoretical treatment of 3PA in zinc-blende semiconductors. The first subsection focuses on the Kane eight-band model for electronic states, and the second part combines the band structure with third-order perturbation theory to calculate 3PA coefficients. Note that we refer to the model as comprising eight bands, while it is sometimes described in the literature as a four-band model. The reason for this becomes apparent in the second part of the appendix; although a given pair of bands may be energy degenerate, they each have their own optical selection rules and therefore must be treated as separate entities.

\section{A. Kane band structure}

We use the band structure model developed by Kane [32] because it includes band symmetry intermixing that is ignored in simplified two-band models. We rederive the model here for reference following the original work and the excellent summary in [62]. We start with the Schrödinger equation in the following form:

$$
H_{0} \Psi_{n}(\mathbf{r})=E_{n} \Psi(\mathbf{r}),
$$

where $H_{0}=\mathbf{p}^{2} /\left(2 m_{0}\right)+V(\mathbf{r})$ is the usual Hamiltonian operator. $\Psi_{n}$ and $E_{n}$ are the wavefunction and energy, respectively, of an electron in an eigenstate labeled by $n$. The symbol $\mathbf{p}$ is the momentum operator, $m_{0}$ is the electron mass, and $V(\mathbf{r})$ is the one-electron potential. Substituting the Bloch form,

$$
\Psi_{n \mathbf{k}}(\mathbf{r})=\exp (i \mathbf{k} \cdot \mathbf{r}) u_{n \mathbf{k}}(\mathbf{r}),
$$

into Eq. (A1) gives the Hamiltonian

$$
H_{0} u_{n \mathbf{k}}(\mathbf{r})+\left(\frac{\hbar}{m}\right) \mathbf{k} \cdot \mathbf{p} u_{n \mathbf{k}}(\mathbf{r})=E_{n}^{\prime} u_{n \mathbf{k}}(\mathbf{r}),
$$

where we have defined

$$
E_{n}^{\prime}=E_{n}-\frac{\hbar^{2} k^{2}}{2 m_{0}}
$$

At the zone center $(\mathbf{k}=0)$, Eq. (A3) reduces to a bare Schrödinger equation for each unit cell function, $u_{n 0}$. We choose a finite set of orthonormal $u_{n 0}$ and assume that they form a complete set, then expand the $u_{n \mathbf{k}}$ of Eq. (A3) in this basis as

$$
u_{n \mathbf{k}}=\sum_{m} c_{m n}(\mathbf{k}) u_{m 0}(\mathbf{r}) .
$$

Note that we could have chosen a basis defined anywhere throughout the Brillouin zone, but the zone center is the most convenient reference because it is the point of highest symmetry. Obviously, the accuracy of the expansion in Eq. (A5) depends on the dimensionality of the basis. The bands mix as the wave vector increases due to interaction from the $\mathbf{k} \cdot \mathbf{p}$ term, which is the origin of the term $\mathbf{k} \cdot \mathbf{p}$ theory. By premultiplying $u_{k 0}(\mathbf{r})$ and integrating over the crystal volume, we can form a matrix equation for the expansion coefficients, $c_{k n}$. This matrix equation can be solved perturbatively or diagonalized exactly. Here, we diagonalize the matrix exactly and ignore perturbative contributions from bands outside the basis. 
We include spin by taking the total unit cell function to be the tensor product of spatial and spin degrees of freedom, then the spin-orbit interaction term

$$
H_{\mathrm{SO}}=\frac{\hbar}{4 m_{0}^{2} c^{2}}(\nabla V \times \mathbf{p}) \cdot \boldsymbol{\sigma},
$$

is added to Eq. (A1). In Eq. (A6), $\sigma$ is the vector of Pauli spin matrices that acts on the spin components, and $c$ is the speed of light. Substituting the Bloch form [Eq. (A2)] into the updated Hamiltonian simplifies to

$$
\begin{aligned}
& {\left[H_{0}+\left(\frac{\hbar}{m}\right) \mathbf{k} \cdot \mathbf{p}+\left(\frac{\hbar}{4 m^{2} c^{2}}\right)(\nabla V \times \mathbf{p}) \cdot \boldsymbol{\sigma}\right]\left|u_{n \mathbf{k}}(\mathbf{r}) ; s\right\rangle} \\
& \quad=E_{n}^{\prime}\left|u_{n \mathbf{k}}(\mathbf{r}) ; s\right\rangle
\end{aligned}
$$

after neglecting k-dependent spin-orbit terms. In Eq. (A7), we separated the spatial and spin degrees of freedom with a semicolon, but henceforth we will represent the spin by an arrow after the spatial component's label.

In the zinc-blende structures studied here, the states closest to the Fermi energy are a doubly spin degenerate $s$-like conduction band and $p$-like valence bands that are six-fold degenerate (including spin) before spin-orbit interaction. While the eight basis functions $|i S \uparrow\rangle,|i S \downarrow\rangle,|X \uparrow\rangle,|X \downarrow\rangle,|Y \uparrow\rangle,|Y \downarrow\rangle,|Z \uparrow\rangle$, and $|Z \downarrow\rangle$ are a perfectly acceptable choice, we find the most convenient Hamiltonian matrix by selecting valence basis functions from the spherical harmonics $Y_{10}=|Z\rangle$ and $Y_{1 \pm 1}=\mp(X \pm i Y) / \sqrt{2}$. Thus, the full ordered basis is taken to be

$$
\begin{aligned}
& |i S \downarrow\rangle,\left|\frac{(X-i Y)}{\sqrt{2}} \uparrow\right\rangle, \quad|Z \downarrow\rangle,\left|\frac{(X+i Y)}{\sqrt{2}} \uparrow\right\rangle, \\
& |i S \uparrow\rangle,\left|-\frac{(X+i Y)}{\sqrt{2}} \downarrow\right\rangle, \quad|Z \uparrow\rangle,\left|\frac{(X-i Y)}{\sqrt{2}} \downarrow\right\rangle .
\end{aligned}
$$

Fixing $\mathbf{k}=k \hat{\mathbf{z}}$, the Hamiltonian matrix is in block diagonal form

$$
\left[\begin{array}{cl}
H & 0 \\
0 & H
\end{array}\right] \quad \text { where } H=\left[\begin{array}{cccc}
E_{s} & 0 & k P & 0 \\
0 & E_{p}-\frac{\Delta}{3} & \frac{\sqrt{2} \Delta}{3} & 0 \\
k P & \frac{\sqrt{2} \Delta}{3} & E_{p} & 0 \\
0 & 0 & 0 & E_{p}+\frac{\Delta}{3}
\end{array}\right] .
$$

$E_{s}$ and $E_{p}$ are the zone center energies defined by $H_{0} u_{j 0}=$ $E_{j} u_{j 0}$. These are chosen to be $E_{s}=E_{g}$ and $E_{p}=-\Delta / 3$ so that the last row and column go to zero. This choice reduces the problem to the diagonalization of two identical $3 \times 3$ matrices. $P$ is the Kane parameter defined by

$$
\begin{aligned}
P & =-i(\hbar / m)\left\langle S\left|p_{z}\right| Z\right\rangle=-i(\hbar / m)\left\langle S\left|p_{y}\right| Y\right\rangle \\
& =-i(\hbar / m)\left\langle S\left|p_{x}\right| X\right\rangle
\end{aligned}
$$

and $\Delta$ is the spin-orbit interaction energy

$$
\Delta=\frac{3 \hbar i}{4 m^{2} c^{2}}\left\langle X\left|\frac{\partial V}{\partial x} p_{y}-\frac{\partial V}{\partial y} p_{x}\right| Y\right\rangle
$$

All equivalent matrix elements can be generated from Eq. (A11) by applying all symmetry operations of the crystal's point group, as was done for $P$ in Eq. (A10).
Diagonalizing Eq. (A9) at the zone center gives eigenstates of the total angular momentum $J=L+S$ (orbital plus spin) and its $z$ component $J_{z}$. The s-like $(L=0)$ conduction band is a doubly degenerate state with $L=0$ and $S= \pm 1 / 2$. The $p$-like $(L=1)$ valence bands include heavy holes with quantum numbers $\left(J, J_{z}\right)=(3 / 2, \pm 3 / 2)$, light holes with $(3 / 2, \pm 1 / 2)$, and splitoff holes with $(1 / 2, \pm 1 / 2)$. Spin-orbit coupling serves to split the $J=1 / 2$ states from $J=3 / 2$ states by energy $\Delta$, hence the name split-off holes.

For arbitrary $\mathbf{k}$, we simply rotate to primed coordinates where $\mathbf{k}=k \hat{\mathbf{z}}^{\prime}$. The Hamiltonian in the new coordinates has the exact same form as Eq. (A7), but with basis functions transformed according to their representation. Because the valence bands belong to $\Gamma_{4}$ which transform as the components of a vector, the transformation takes the form of the usual vector rotation matrix

$$
\left[\begin{array}{l}
X^{\prime} \\
Y^{\prime} \\
Z^{\prime}
\end{array}\right]=\left[\begin{array}{ccc}
\cos \theta \cos \phi & \cos \theta \sin \phi & -\sin \theta \\
-\sin \phi & \cos \phi & 0 \\
\sin \theta \cos \phi & \sin \theta \sin \phi & \cos \theta
\end{array}\right]\left[\begin{array}{l}
X \\
Y \\
Z
\end{array}\right] .
$$

The spin components transform as the two-dimensional irreducible representation of $S U(2)$,

$$
\left[\begin{array}{l}
\uparrow^{\prime} \\
\downarrow^{\prime}
\end{array}\right]=\left[\begin{array}{ll}
e^{-\phi / 2} \cos (\theta / 2) & e^{\phi / 2} \sin (\theta / 2) \\
e^{-\phi / 2} \sin (\theta / 2) & e^{\phi / 2} \cos (\theta / 2)
\end{array}\right]\left[\begin{array}{l}
\uparrow \\
\downarrow
\end{array}\right],
$$

but this rotation is not necessary to compute because the optical selection rule remains unchanged by rotation. Because the conduction bands belong to the identity (spherical) representation $\Gamma_{1}, S^{\prime}=S$ is invariant under rotation. The angles $\theta$ and $\phi$ are the usual polar angles of the $\mathbf{k}$ vector relative to the crystal axes labeled $x, y$ and $z$. The characteristic equation yields four double roots corresponding to the eigenvalues of Eq. (A9):

$$
\begin{aligned}
& E^{\prime}=0 \\
& E^{\prime}\left(E^{\prime}-E_{G}\right)\left(E^{\prime}+\Delta\right)-k^{2} P^{2}\left(E^{\prime}+2 \Delta / 3\right)=0,
\end{aligned}
$$

where $E_{k}=E_{k}^{\prime}+\left(\hbar^{2} / 2 m_{0}\right) k^{2}$ is the energy of a state with a wavevector $\mathbf{k}$. The electronic wave functions are found to be

$$
\begin{aligned}
& u_{i \alpha}=a_{i}[i S \downarrow]^{\prime}+b_{i}[(X-i Y) \uparrow / \sqrt{2}]^{\prime}+c_{i}[Z \downarrow]^{\prime} \\
& u_{i \beta}=a_{i}[i S \uparrow]^{\prime}+b_{i}[-(X+i Y) \downarrow / \sqrt{2}]^{\prime}+c_{i}[Z \uparrow]^{\prime} \\
& u_{\mathrm{HH} \alpha}=[(X+i Y) \uparrow / \sqrt{2}]^{\prime} \\
& u_{\mathrm{HH} \beta}=[(X-i Y) \downarrow / \sqrt{2}]^{\prime},
\end{aligned}
$$

where $\alpha$ and $\beta$ denote the two degenerate spin states, and the index $i$ refers to the conduction, $\mathrm{LH}$, and SO bands. The real coefficients $a, b$, and $c$ are obtained from

$$
\begin{aligned}
& a_{i}=k P\left(E_{i}^{\prime}+2 \Delta / 3\right) / N \\
& b_{i}=(\sqrt{2} \Delta / 3)\left(E_{i}^{\prime}-E_{G}\right) / N \\
& c_{i}=\left(E_{i}^{\prime}-E_{G}\right)\left(E_{i}^{\prime}+2 \Delta / 3\right) / N
\end{aligned}
$$

where $N$ is a normalizing factor such that $a_{i}^{2}+b_{i}^{2}+c_{i}^{2}=1$. 


\section{B. 3PA Coefficients}

Here, we use the results of the previous subsection to compute 3PA coefficients in third-order perturbation theory. We begin with the electron-radiation interaction Hamiltonian in SI units $[63,64]$

$$
H_{p}=\frac{e}{i \omega m_{0}}\left(\frac{I}{2 \varepsilon_{0} n_{0} c}\right)^{1 / 2} \hat{\mathbf{a}} \cdot \hat{\mathbf{p}}
$$

where $I$ is the irradiance of the incident beam, and $n_{0}$ is the material's index of refraction. In the determination of the interaction Hamiltonian, the matrix elements $e /\left(m_{0} c\right) \mathbf{A} \cdot \mathbf{P}$ are needed, which are obtained from the $\mathbf{k} \cdot \mathbf{p}$ theory discussed earlier. Here, $\mathbf{A}$ is the magnetic vector potential, and $\hat{\mathbf{a}}$ is a unit vector parallel to the direction of the incident electric field. We can then express the total 3PA transition rate per unit volume using Fermi's golden rule derived from third-order perturbation theory:

$$
\begin{aligned}
W_{3}= & \frac{2 \pi}{V \hbar} \sum_{c, v} \sum_{\mathbf{k}}\left|\sum_{i, j} \frac{\left\langle c\left|H_{p}\right| j\right\rangle^{\prime}\left\langle j\left|H_{p}\right| i\right\rangle^{\prime}\left\langle I\left|H_{p}\right| v\right\rangle^{\prime}}{\left(E_{j v}(\mathbf{k})-2 \hbar \omega\right)\left(E_{i v}(\mathbf{k})-\hbar \omega\right)}\right|^{2} \\
& \times \delta\left[E_{c v}(\mathbf{k})-3 \hbar \omega\right],
\end{aligned}
$$

with $E_{m n}:=E_{m}-E_{n}$. The index $v$ corresponds to a valence band (initial state), $c$ is a conduction band (final state), and $i$ and $j$ are intermediate states chosen from any of the eight bands detailed in the previous subsection. The kets $|l\rangle$ (and bras $\langle l|$ ) are shorthand for the $\mathbf{k}$-dependent unit cell functions, $u_{l \mathbf{k}}$, as calculated by Eqs. (A15) and (A16); the plane wave portion of the Bloch state [Eq. (A2)] has already been used to enforce the crystal momentum selection rule leaving a single sum over wave vector $\mathbf{k}$. Primes on the interaction matrix elements indicate that basis functions are rotated according to the transformation in Eq. (A12). Note that quantum interference occurs due to the intermediate state sum within the absolute value brackets for a given $c, v$, and $\mathbf{k}$. Because each term in this sum can have arbitrary sign and magnitude, the net contribution to 3PA can only be determined by adding up the effect of all possible paths. We also note that, because application of the Pauli exclusion principle does not affect the outcome of Eq. (A18), we can ignore it in our intermediate state sum calculations $[65,66]$.

The transition rate per unit volume is used to calculate the 3PA coefficient by relating electron transition rate to photon flux with $[20,67]$ :

$$
\alpha_{3}(\omega)=\frac{3 \hbar \omega W_{3}}{I^{3}} .
$$

Combining Eqs. (A17)-(A19) yields the final expression for 3PA coefficients for z-polarized light:

$$
\begin{aligned}
\alpha_{3} & =3 \omega \frac{(2 \pi)^{5}}{\left(n_{0} c\right)^{3}}\left(\frac{e P}{\hbar \omega}\right)^{6} \sum_{c, v} \int_{0}^{\pi} \\
& \left(\left|\sum_{i, j} \frac{M_{c j}^{z}\left(k_{r}, \theta\right) M_{j i}^{z}\left(k_{r}, \theta\right) M_{i v}^{z}\left(k_{r}, \theta\right)}{\left(E_{i v}\left(k_{r}\right)-\hbar \omega\right)\left(E_{j v}\left(k_{r}\right)-2 \hbar \omega\right)}\right|^{2}\right) \frac{k_{r}^{2} \sin \theta \mathrm{d} \theta}{\left|\frac{\partial E_{c v}(k)}{\partial k}\right|_{k=k_{r}}},
\end{aligned}
$$

where

$$
M_{i j}^{z}\left(k_{r}, \theta\right)=3 \frac{\hbar}{m_{0} P}\left\langle u_{i}\left(k_{r}, \theta\right)\left|p_{z}\right| u_{j}\left(k_{r}, \theta\right)\right\rangle^{\prime},
$$

are the normalized momentum matrix elements [55]. Note that the $\mathbf{k}$ sum in Eq. (A18) has been converted to a volume integral by $(1 / V) \sum_{k} \rightarrow \int(2 \pi)^{3} \mathrm{~d}^{3} \mathbf{k}$ and evaluated in spherical coordinates. The remaining $\delta$-function integration results in an integral over constant energy surfaces defined by

$$
E_{c v}\left(k_{r}\right)-3 \hbar \omega=0,
$$

with $k_{r}$ denoting the $k$ value for which the transition is resonant.

The $\mathrm{z}$ direction was chosen so that matrix elements are independent of azimuthal angle. Inspection of the third column of the rotation matrix Eq. (A12) shows that the $Z$ component of rotated basis functions only depends on angle $\theta$. Because the only nonzero matrix element for $\mathrm{z}$-polarized light is of the form $\left\langle i S\left|p_{z}\right| Z\right\rangle$, the matrix elements only depend on $\theta$, while the $\phi$ integral evaluates to $2 \pi$. We chose z polarization for convenience, but analysis for light polarized in any direction would yield the same result due to the spherical symmetry of the Kane band structure.

\section{APPENDIX B: SPIN-ORBIT COUPLING AND QUANTUM INTEREFRENCE IN 3PA SPECTRA}

This appendix aims to provide insight into the shapes and parameter dependence of the curves in Fig. 5. Given the large number of possible 3PA pathways, it is not immediately obvious which terms dominate at a given three-photon energy sum. While it is possible to write each term individually and compare the magnitudes at a given point in $k$ space, it is just as illuminating to give a semiqualitative reason for the dispersions shown. We begin by carefully examining the case with no spin-orbit coupling and demonstrate how the complexity in the spectrum increases with this coupling.

The conceptually simplest description exists for materials with weak spin-orbit coupling: AlN, $\mathrm{ZnO}$, and GaN. We study these by assuming $\Delta=0$ so that only $\mathbf{k} \cdot \mathbf{p}$ coupling terms between $|S\rangle^{\prime}$ and $|Z\rangle^{\prime}$ remain in the Hamiltonian matrix (Eq. A9). We are free to rearrange the order of the basis to group functions of the same spin into a single block. Without loss of generality, we diagonalize the spin up block to find

$$
\begin{aligned}
& u_{C}=a_{c}(k)|i S\rangle^{\prime}+b_{B}(k)|Z\rangle^{\prime} \\
& u_{\mathrm{SO}}=a_{z}(k)|i S\rangle^{\prime}+b_{z}(k)|Z\rangle^{\prime} \\
& u_{\mathrm{HH}}=\frac{1}{\sqrt{2}}|X-i Y\rangle^{\prime} \\
& u_{\mathrm{LH}}=\frac{1}{\sqrt{2}}|X+i Y\rangle^{\prime} .
\end{aligned}
$$

The conduction and SO bands intermix, while the $\mathrm{HH}$ and $\mathrm{LH}$ bands remain uncoupled and retain a free-electron-like dispersion. We compute $z$ matrix elements by rotating the primed basis back to unprimed functions using Eq. (A12). Then, applying Eq. (A10) and normalizing by Eq. (A21) yields

$$
\begin{aligned}
& M_{C-\mathrm{HH}}^{z}=\left\langle u_{c}\left|p_{z}\right| u_{\mathrm{HH}}\right\rangle=\frac{3}{\sqrt{2}} a_{c} \sin \theta \\
& M_{C-\mathrm{LH}}^{z}=\left\langle u_{\mathrm{c}}\left|p_{z}\right| u_{\mathrm{LH}}\right\rangle=\frac{3}{\sqrt{2}} a_{c} \sin \theta .
\end{aligned}
$$

The matrix elements are identical because $\left\langle i S\left|p_{z}\right| Z\right\rangle$ is the only nonzero matrix element by symmetry, and only the $|X\rangle^{\prime}$ term contains a $|Z\rangle$ component when rotated into the unprimed 
coordinates. The same behavior exists for $M_{c-\text { so }}$; the $|Z\rangle$ couples with the $|i S\rangle$ component of $u_{\mathrm{so}}$. Because these bands experience the same optical coupling and are energy degenerate throughout the Brillouin zone, contributions to the 3PA coefficient from transitions originating in $\mathrm{LH}$ and $\mathrm{HH}$ bands will be identical. As we mentioned in the main text, for brevity we refer to these as the $\mathrm{HH}$ and $\mathrm{LH}$ contributions. By comparing the red and blue curves in Fig. 5 for $\mathrm{AlN}, \mathrm{ZnO}$, and $\mathrm{GaN}$, we see the equality of these contributions except for minor difference because $\Delta$ is not exactly zero.

We reasonably approximate the shape of these curves with low spin-orbit coupling by the expression

$$
\alpha_{3} \propto \frac{1}{x^{5}}\left|\frac{A}{x^{2}}+\frac{B}{x^{2}}(3 x-1)\right|^{2}(3 x-1)^{\frac{1}{2}}
$$

where $x$ is the photon energy normalized to the bandgap, $\hbar \omega / E_{g}$. Eq. (B3) is derived from Eq. (A20) by first solving for $k_{r}$ using the approximate energy conservation condition

$$
E_{c v}\left(k_{r}\right)-3 \hbar \omega \approx E_{g}+\frac{\hbar^{2} k_{r}^{2}}{2 \mu_{c v}}-3 \hbar \omega=0,
$$

where $1 / \mu_{c v}=\left(1 / \mu_{c}-1 / \mu_{v}\right)$ is the inverse reduced effective mass. Normalizing all energies, we obtain

$$
k_{r}=\frac{\sqrt{2 \mu_{c v}}}{\hbar}(3 x-1)^{1 / 2}
$$

The $A$ term in Eq. (B3) describes triply-allowed 3PA pathways with $\mathbf{k}$-independent matrix elements. The $B$ term accounts for allowed-forbidden-forbidden transitions and picks up a $k_{r}^{2}$ term because each forbidden matrix element is approximately linear in $k$ near the zone center.

Quantum interference arises due to differing relative magnitudes and signs between $A$ and $B$ in Eq. (B3). The triply-allowed term dominates for $\mathrm{HH}$ and $\mathrm{LH}$ contributions when $\Delta \approx 0$, leading to a 3PA which scales as Wherrett's theory predicts [Eq. (2)]. From this, we determine that there is either constructive or weak destructive interference, and the actual result can only be found by tediously expanding each term in the sum and comparing. In contrast to the $\mathrm{HH}$ and $\mathrm{LH}$, the SO contribution clearly demonstrates strong deconstructive interference: the triply-allowed term still dominates near the zone center, but the allowed-forbiddenforbidden terms have opposite sign and grow as $k_{r}$ increases. Eventually this term grows larger than the triply-allowed term, flipping the sign of the sum. Because the 3PA coefficient scales with the magnitude squared of the sum, this sign change appears as a drop down to zero followed by a bounce back upward. This is very clearly seen in the green curve for $\mathrm{ZnO}$.

As spin-orbit splitting increases from zero, the $\mathrm{LH}$ wave function becomes distinguished from that of the $\mathrm{HH}$. However, this identity only persists throughout a region of the Brillouin zone determined by the strength of the spin-orbit interaction. Quantitatively, this means that $a_{\mathrm{LH}}$ and $c_{\mathrm{LH}}$ of Eq. (A15) decay away to zero as $k$ increases so that the wave function tends to the same form as that for $\mathrm{HH}$. When spin-orbit coupling is small, this decrease occurs very quickly. See Fig. 7 for a plot of the expansion coefficients: Eq. A1(a) shows how the wave functions change throughout the Brillouin zone in $\operatorname{InP}\left(\Delta / E_{g}=0.08\right)$, and Eq. A1(b) shows what happens when $\Delta / E_{g}=0.008$, near to the value for GaN. Figure 8(a) shows the band structure obtained for
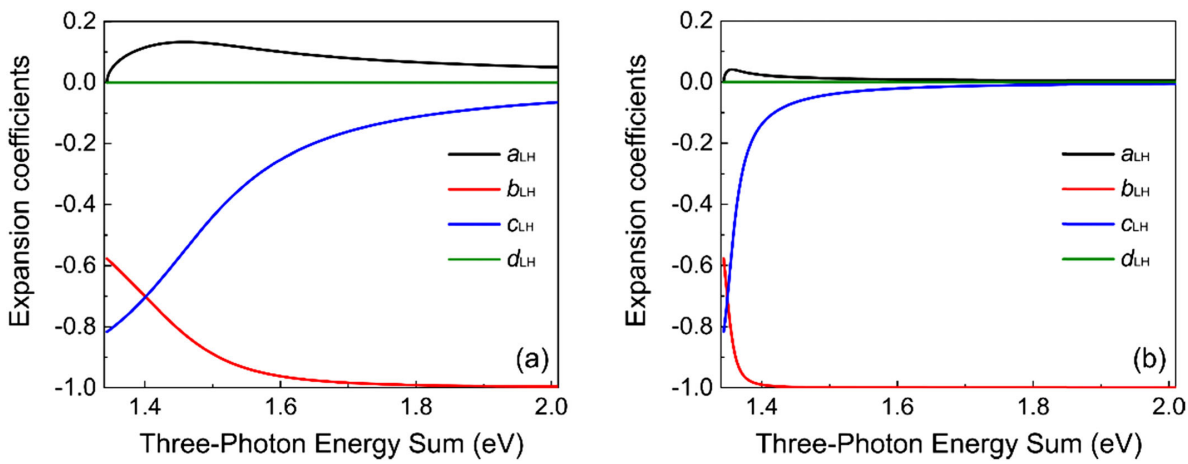

Fig. 7. Expansion coefficients for the light hole unit cell function versus three-photon energy sum for simulated values of (a) $\Delta / E_{g}=0.08$ and (b) $\Delta / E_{g}=0.008$. The horizontal axis is related to $k$ by $E_{c v}(k)=3 \hbar \omega$ for $v=L H$.
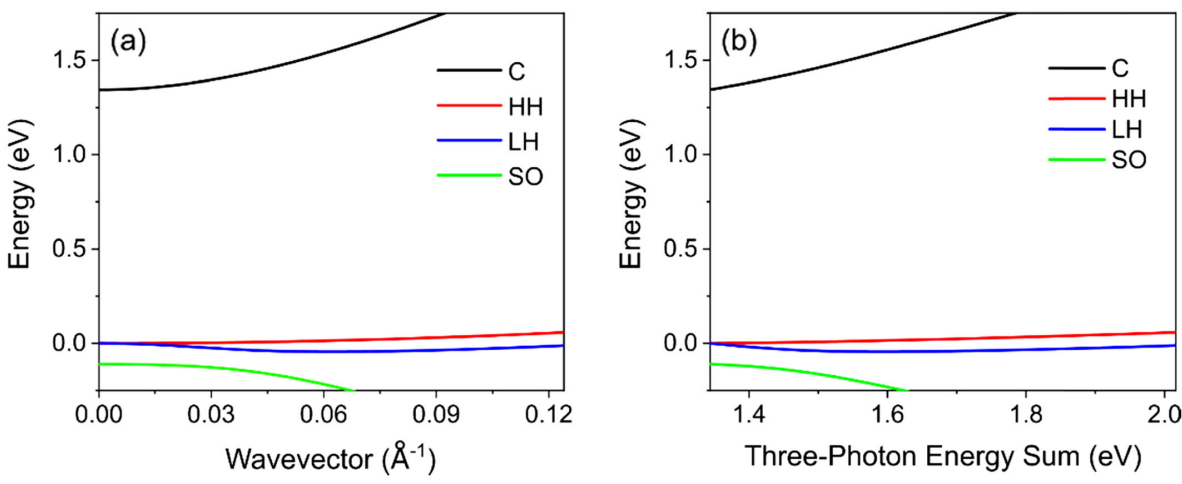

Fig. 8. Energy bands of InP calculated from parameters given in Table 2 with horizontal axes chosen to be (a) $k$ and (b) three-photon energy sum resonant with the conduction (C) to LH gap at a given $k\left(E_{c v}(k)=3 \hbar \omega\right.$ with $\left.v=L H\right)$. The ranges of $k$ values in (a) and (b) are identical. 
InP. To facilitate easy comparison with features in Fig. 5, we also plot in Fig. 8(b) the same band structure but with the horizontal axis to be the three-photon energy sum resonant with a light hole to conduction transitions. Specifically, three-photon energy sums correspond to a given $k$ by the relation $E_{c v}(k)=3 \hbar \omega$ where $v=\mathrm{LH}$. Figure 8(a) shows that the slopes of $\mathrm{HH}$ and $\mathrm{LH}$ band energies become nearly identical at large $k$.

For intermediate $\Delta / E_{g}$, we see that the LH and $\mathrm{HH}$ contributions in Fig. 5 nearly equalize at some photon energy. Some minor differences may remain, attributable to differing denominators in the perturbation expansion. This merging of $\mathrm{LH}$ and $\mathrm{HH}$ contributions is apparent in the 3PA curves for InP, AlP, $\mathrm{ZnS}$, and CdS. Comparing Fig. 7 and Fig. 5, we see that $\mathrm{HH}$ and $\mathrm{LH}$ 3PA contributions approach each other as the magnitude of the $\mathrm{HH}$-like expansion coefficient $\left(\left|b_{\mathrm{LH}}\right|\right)$ of the LH band nears unity.

LH contributions for intermediate $\Delta / E_{g}$ still experience destructive interference as the triply-allowed term is partially cancelled by allowed-forbidden-forbidden transitions. Note, however, that this cancellation is more complex than that of SO holes with $\Delta=0$. As shown in Fig. 7 , the $|i S\rangle$ component of the LH wavefunction increases then decreases, leading to nonmonotonic forbidden transition matrix elements that depend on $a_{\mathrm{LH}}$. This nonmonotonicity partially explains why the interference does not lead the 3PA coefficient to pass through 0 as in the $\Delta=0$ limit. For materials with large spin-orbit coupling, the $\mathrm{LH}$ and $\mathrm{HH}$ curves remain distinct throughout the entire region of interest, and the 3PA spectral shape is not easily attributed to a specific interaction term. This is the behavior exhibited by CdTe, GaSb, InAs, and InSb.

Funding. National Science Foundation (DMR-1609895).

Acknowledgment. We thank Kirk Scammon of Materials Characterization Facility AMPAC at the University of Central Florida for helping us with determining the crystal orientation of our samples.

Disclosures. The authors declare no conflicts of interest.

\section{REFERENCES}

1. M. Chattopadhyay, P. Kumbhakar, R. Sarkar, and A. Mitra, "Enhanced three-photon absorption and nonlinear refraction in $\mathrm{ZnS}$ and $\mathrm{Mn}^{2+}$ doped ZnS quantum dots," Appl. Phys. Lett. 95, 163115 (2009).

2. G. S. He, J. D. Bhawalkar, P. N. Prasad, and B. A. Reinhardt, "Threephoton-absorption-induced fluorescence and optical limiting effects in an organic compound," Opt. Lett. 20, 1524-1526 (1995).

3. G. S. He, P. P. Markowicz, T.-C. Lin, and P. N. Prasad, "Observation of stimulated emission by direct three-photon excitation," Nature $\mathbf{4 1 5}$, 767-770 (2002).

4. G. S. He, L.-S. Tan, Q. Zheng, and P. N. Prasad, "Multiphoton absorbing materials: molecular designs, characterizations, and applications," Chem. Rev. 108, 1245-1330 (2008).

5. J. He, Y. Qu, H. Li, J. Mi, and W. Ji, "Three-photon absorption in $\mathrm{ZnO}$ and ZnS crystals," Opt. Express 13, 9235-9247 (2005).

6. W. C. Hurlbut, Y.-S. Lee, K. Vodopyanov, P. Kuo, and M. Fejer, "Multiphoton absorption and nonlinear refraction of GaAs in the mid-infrared," Opt. Lett. 32, 668-670 (2007).

7. S. Pearl, N. Rotenberg, and H. M. van Driel, "Three photon absorption in silicon for 2300-3300 nm," Appl. Phys. Lett. 93, 131102 (2008).

8. P. Zhao, M. Reichert, D. J. Hagan, and E. W. Van Stryland, "Dispersion of nondegenerate nonlinear refraction in semiconductors," Opt. Express 24, 24907-24920 (2016)
9. G. Polónyi, B. Monoszlai, G. Gäumann, E. J. Rohwer, G. Andriukaitis, T. Balciunas, A. Pugzlys, A. Baltuska, T. Feurer, and J. Hebling, "Highenergy terahertz pulses from semiconductors pumped beyond the three-photon absorption edge," Opt. Express 24, 23872-23882 (2016).

10. M. R. Shcherbakov, K. Werner, Z. Fan, N. Talisa, E. Chowdhury, and G. Shvets, "Photon acceleration and tunable broadband harmonics generation in nonlinear time-dependent metasurfaces," Nat. Commun. 10, 1345 (2019).

11. M. Reichert, A. L. Smirl, G. Salamo, D. J. Hagan, and E. W. Van Stryland, "Observation of nondegenerate two-photon gain in GaAs," Phys. Rev. Lett. 117, 073602 (2016).

12. M. Reichert, P. Zhao, H. S. Pattanaik, D. J. Hagan, and E. W. Van Stryland, "Nondegenerate two-and three-photon nonlinearities in semiconductors," Proc. SPIE 9835, 98350A (2016).

13. J. He, W. Ji, J. Mi, Y. Zheng, and J. Y. Ying, "Three-photon absorption in water-soluble ZnS nanocrystals," Appl. Phys. Lett. 88, 181114 (2006).

14. A. D. Lad, P. Prem Kiran, G. Ravindra Kumar, and S. Mahamuni, "Threephoton absorption in ZnSe and Zn Se/Zn S quantum dots," Appl. Phys. Lett. 90, 133113 (2007).

15. Y. Wang, V. D. Ta, Y. Gao, T. C. He, R. Chen, E. Mutlugun, H. V. Demir, and H. D. Sun, "Stimulated emission and lasing from CdSe/CdS/ZnS coremulti-shell quantum dots by simultaneous three-photon absorption," Adv. Mater. 26, 2954-2961 (2014).

16. S. S. Mitra, N. Judell, A. Vaidyanathan, and A. H. Guenther, "Threephoton absorption in direct-gap crystals," Opt. Lett. 7, 307-309 (1982).

17. Z. Wang, H. Liu, N. Huang, Q. Sun, J. Wen, and X. Li, "Influence of three-photon absorption on mid-infrared cross-phase modulation in silicon-on-sapphire waveguides," Opt. Express 21, 1840-1848 (2013).

18. C. Husko, S. Combrié, Q. Tran, F. Raineri, A. De Rossi, and C. Wong, "Slow-light enhanced self-phase modulation, three-photon absorption and free-carriers in photonic crystals: experiment and theory," in Laser Science to Photonic Applications (CLEO/QELS) (IEEE, 2010), pp. 1-2.

19. E. W. Van Stryland, H. Vanherzeele, M. A. Woodall, M. Soileau, A. L. Smirl, S. Guha, and T. F. Boggess, "Two photon absorption, nonlinear refraction, and optical limiting in semiconductors," Opt. Eng. 24, 244613 (1985).

20. B. Wherrett, "Scaling rules for multiphoton interband absorption in semiconductors," J. Opt. Soc. Am. B 1, 67-72 (1984).

21. E. W. Van Stryland, M. Woodall, H. Vanherzeele, and M. Soileau, "Energy band-gap dependence of two-photon absorption," Opt. Lett. 10, 490-492 (1985).

22. J. H. Yee, "Three-photon absorption in semiconductors," Phys. Rev. B 5, 449 (1972).

23. A. Vaidyanathan, T. Walker, A. Guenther, S. Mitra, and L. Narducci, "Twophoton absorption in several direct-gap crystals," Phys. Rev. B 21, 743 (1980).

24. A. Vaidyanathan, A. Guenther, and S. Mitra, "Two-photon absorption in direct-gap crystals - an addendum," in Laser Induced Damage In Optical Materials: 1980 (ASTM International, 1981).

25. J. M. Hales, S.-H. Chi, T. Allen, S. Benis, N. Munera, J. W. Perry, D. McMorrow, D. J. Hagan, and E. W. Van Stryland, "Third-order nonlinear optical coefficients of $\mathrm{Si}$ and GaAs in the near-infrared spectral region," in Applications and Technology (CLEO) (Optical Society of America, 2018), paper JTu2A. 59.

26. D. Hutchings and B. Wherrett, "Linear/circular dichroism of two-photon absorption in zinc-blende semiconductors," Opt. Mater. 3, 53-60 (1994).

27. D. Hutchings and B. Wherrett, "Theory of anisotropy of two-photon absorption in zinc-blende semiconductors," Phys. Rev. B 49, 2418 (1994).

28. L. V. Keldysh, “Zh. É ksp. Teor. Fiz. 47, 19451964 Sov. Phys,” JETP 20, 1307 (1965).

29. C. M. Cirloganu, P. D. Olszak, L. A. Padilha, S. Webster, D. J. Hagan, and E. W. Van Stryland, "Three-photon absorption spectra of zinc blende semiconductors: theory and experiment," Opt. Lett. 33, 2626-2628 (2008).

30. C. M. Cirloganu, P. D. Olszak, L. A. Padilha, S. Webster, D. J. Hagan, and E. W. Van Stryland, "Three-photon absorption spectra of zinc blende semiconductors: theory and experiment: erratum," Opt. Lett. 45, 1025-1026 (2020)

31. H. Brandi and C. De Araujos, "Multiphonon absorption coefficients in solids: a universal curve," J. Phys. C 16, 5929 (1983). 
32. E. O. Kane, "Band structure of indium antimonide," J. Phys. Chem. Solids 1, 249-261 (1957).

33. M. Hasselbeck, A. Said, E. Van Stryland, and M. Sheik-Bahae, "Threephoton absorption in InAs," Opt. Quantum Electron. 30, 193-200 (1998).

34. J. U. Kang, A. Villeneuve, M. Sheik-Bahae, G. I. Stegeman, K. AlHemyari, J. S. Aitchison, and C. N. Ironside, "Limitation due to three-photon absorption on the useful spectral range for nonlinear optics in AlGaAs below half band gap," Appl. Phys. Lett. 65, 147-149 (1994).

35. G.-M. Schucan, R. G. Ispasoiu, A. M. Fox, and J. F. Ryan, "Ultrafast two-photon nonlinearities in CdSe near $1.5 / \mathrm{spl} \mathrm{mu/m}$ studied by interferometric autocorrelation," IEEE J. Quantum Electron. 34, 1374-1379 (1998).

36. M. Sheik-Bahaei, P. Mukherjee, and H.-S. Kwok, "Two-photon and three-photon absorption coefficients of InSb," J. Opt. Soc. Am. B 3, 379-385 (1986).

37. J. Bechtel and W. L. Smith, "Two-photon absorption in semiconductors with picosecond laser pulses," Phys. Rev. B 13, 3515 (1976).

38. M. Sheik-Bahae, A. A. Said, T.-H. Wei, D. J. Hagan, and E. W. Van Stryland, "Sensitive measurement of optical nonlinearities using a single beam," IEEE J. Quantum Electron. 26, 760-769 (1990).

39. M. Sheik-Bahae, A. A. Said, and E. W. Van Stryland, "High-sensitivity, single-beam n 2 measurements," Opt. Lett. 14, 955-957 (1989).

40. S. Shabahang, G. Tao, M. P. Marquez, H. Hu, T. R. Ensley, P. J. Delfyett, and A. F. Abouraddy, "Nonlinear characterization of robust multimaterial chalcogenide nanotapers for infrared supercontinuum generation," J. Opt. Soc. Am. B 31, 450-457 (2014).

41. M. A. Woodall, Nonlinear Absorption Techniques and Measurements in Semiconductors (1985).

42. S. Guha, E. W. Van Stryland, and M. Soileau, "Self-defocusing in CdSe induced by charge carriers created by two-photon absorption: erratum," Opt. Lett. 11, 59 (1986).

43. S. Guha, E. W. Van Stryland, and M. Soileau, "Self-defocusing in CdSe induced by charge carriers created by two-photon absorption," Opt. Lett. 10, 285-287 (1985).

44. P. D. Olszak, C. M. Cirloganu, S. Webster, L. A. Padilha, S. Guha, L. P. Gonzalez, S. Krishnamurthy, D. J. Hagan, and E. W. Van Stryland, "Spectral and temperature dependence of two-photon and free-carrier absorption in InSb," Phys. Rev. B 82, 235207 (2010).

45. S. Z. Karazhanov and L. L. Y. Voon, "Ab initio studies of the band parameters of III-V and II-VI zinc-blende semiconductors," Semiconductors 39, 161-173 (2005).

46. S. Krishnamurthy, Z. G. Yu, L. P. Gonzalez, and S. Guha, "Temperatureand wavelength-dependent two-photon and free-carrier absorption in GaAs, InP, GalnAs, and InAsP," J. Appl. Phys. 109, 033102 (2011).

47. I. Vurgaftman, J. Meyer, and L. Ram-Mohan, "Band parameters for III-V compound semiconductors and their alloys," J. Appl. Phys. 89, 5815-5875 (2001).
48. B. V. Olson, M. P. Gehlsen, and T. F. Boggess, "Nondegenerate two-photon absorption in GaSb," Opt. Commun. 304, 54-57 (2013).

49. J. Wei, J. Murray, C. Reyner, and S. Guha, "Measurement of Wavelength and Temperature Dependent Refractive Index of GaSb," in Novel Optical Materials and Applications (Optical Society of America, 2019), paper NoM3B. 3.

50. M. Cardona, K. L. Shaklee, and F. H. Pollak, "Electroreflectance at a semiconductor-electrolyte interface," Phys. Rev. 154, 696 (1967).

51. P. Rochon and E. Fortin, "Photovoltaic effect and interband magneto-optical transitions in InP," Phys. Rev. B 12, 5803 (1975).

52. P. Zory, Jr., Quantum Well Lasers (Academic, 1993).

53. M. Cardona and G. Harbeke, "Optical properties and band structure of wurtzite-type crystals and rutile," Phys. Rev. 137, A1467 (1965).

54. M. Willatzen, M. Cardona, and N. Christensen, "Spin-orbit coupling parameters and electron g factor of II-VI zinc-blende materials," Phys. Rev. B 51, 17992 (1995).

55. D. Hutchings and E. W. Van Stryland, "Nondegenerate two-photon absorption in zinc blende semiconductors," J. Opt. Soc. Am. B 9, 2065-2074 (1992)

56. D. Thomas, "The exciton spectrum of zinc oxide," J. Phys. Chem. Solids 15, 86-96 (1960).

57. Y. S. Ang and C. Zhang, "Step-like multi-photon absorption in twodimensional semiconductors with Rashba spin-orbit coupling in terahertz regime," in 39th International Conference on Infrared, Millimeter, and Terahertz waves (IRMMW-THz) (IEEE, 2014), p. 1.

58. X. Feng, Y. L. Ang, J. He, C. W. Beh, H. Xu, W. S. Chin, and W. Ji, "Threephoton absorption in semiconductor quantum dots: experiment," Opt. Express 16, 6999-7005 (2008).

59. A. Mang and K. Reimann, "Band gaps, crystal-field splitting, spinorbit coupling, and exciton binding energies in $\mathrm{ZnO}$ under hydrostatic pressure," Solid State Commun. 94, 251-254 (1995).

60. P. Lambropoulos and M. Teague, "Two-photon ionization with spin-orbit coupling," J. Phys. B 9, 587 (1976).

61. J. H. Davies, The Physics of Low-Dimensional Semiconductors: An Introduction (Cambridge University, 1998).

62. S. L. Chuang and S. L. Chuang, Physics of Optoelectronic Devices (1995).

63. S. M. Sze and K. K. Ng, Physics of Semiconductor Devices (Wiley, 2006).

64. M. Dinu, "Dispersion of phonon-assisted nonresonant third-order nonlinearities," IEEE J. Quantum Electron. 39, 1498-1503 (2003).

65. M. Reichert, Nonlinear Optical Response of Simple Molecules and TwoPhoton Semiconductor Lasers (2015).

66. S. L. Chuang, Physics of Photonic Devices (Wiley, 2012), Vol. 80.

67. R. W. Boyd, Nonlinear optics (Elsevier, 2003). 